\title{
Robust expression of tumor suppressor miRNA's let-7 and miR-195 detected in plasma of Saudi female breast cancer patients
}

Amal Qattan ${ }^{1,2,3^{*}}$ (D, Haya Intabli ${ }^{1,3}$, Wafa Alkhayal ${ }^{4,5}$, Chafica Eltabache ${ }^{1}$, Taher Tweigieri ${ }^{6}$ and Suad Bin Amer ${ }^{1^{*}}$

\begin{abstract}
Background: Female breast cancer is frequently diagnosed at a later stage and the leading cause of cancer deaths world-wide. Levels of cell-free circulating microRNAs (miRNAs) can potentially be used as biomarkers to measure disease progression in breast cancer patients in a non-invasive way and are therefore of high clinical value.

Methods: Using quantitative RT-PCR, circulating miRNAs were measured in blood samples collected from diseasefree individuals $(n=34)$, triple-negative breast tumours (TNBC) $(n=36)$ and luminal tumours $(n=57)$. In addition to intergroup comparisons, plasma miRNA expression levels of all groups were analyzed against RNASeq data from cancerous breast tissue via The Cancer Genome Atlas (TCGA).

Results: A differential set of 18 miRNAs were identified in the plasma of breast cancer patients and 10 miRNAs were uniquely identified based on ROC analysis. The most striking findings revealed elevated tumor suppressor let-7 miRNA in luminal breast cancer patients, irrespective of subtype, and elevated miR-195 in plasma of TNBC breast cancer patients. In contrast, hsa-miR-195 and let-7 miRNAs were absent from cancerous TCGA tissue and strongly expressed in surrounding non-tumor tissue indicating that cancerous cells may selectively export tumor suppressor hsa-miR-195 and let-7 miRNAs in order to maintain oncogenesis.

Conclusions: While studies have indicated that the restoration of let-7 and miR-195 may be a potential therapy for cancer, these results suggested that tumor cells may selectively export hsa-miR-195 and let-7 miRNAs thereby neutralizing their potential therapeutic effect. However, in order to facilitate earlier detection of breast cancer, blood based screening of hsa-miR-195 and let-7 may be beneficial in a female patient cohort.
\end{abstract}

Keywords: Circulating miRNAs, Triple-negative breast cancer (TNBC), Circulating biomarkers, Plasma versus tissue, Secretion, FASN pathway, ROC curves, Cancer therapy

\section{Background}

The general consensus for breast cancer prevention and treatment includes periodic breast cancer screening of all women and the frequent monitoring of women at higher risk [1]. Nevertheless, cancer statistics indicate that as of January 2017 female breast cancer is the most frequently diagnosed cancer [2]. Mammography is the

\footnotetext{
* Correspondence: akattan@kfshrc.edu.sa; aqattan5@gwu.edu; suad@kfshrc.edu.sa

${ }^{1}$ Breast Cancer Research, Department of Molecular Oncology, King Faisal Specialist Hospital and Research Centre, P.O.Box 3354, Riyadh 11211, Saudi Arabia

Full list of author information is available at the end of the article
}

current gold standard for breast cancer screening and is associated with significant discomfort which impedes early detection [3]. Therefore, finding non-invasive, safe, relatively inexpensive and accurate breast cancer tumor markers [4] as well as potential blood-based biomarkers for the diagnosis and prognostics of breast cancer [5-9] remain important research objectives.

MicroRNAs (miRNAs) are short non-coding RNAs which function as post-transcriptional regulators of gene expression through targeted binding [10-12]. While tissue biomarkers have been extensively studied in cancer detection, circulating miRNAs in body fluids, especially blood 
serum and plasma, are a promising source of stable non-invasive biomarkers [13, 14]. The tumor specific de-regulation of some miRNAs and their target genes is frequently observed $[15,16]$ rendering some miRNAs as potential biomarkers for the diagnosis of cancer $[13,17,18]$.

Despite the increase in the number of breast cancer biomarker studies, due to the inconsistency of the results, no consensus has been reached on the diagnostic use of differentially regulated circulating miRNAs reported so far [19]. Inconsistencies among studies may be due to patient heterogeneity; genetic background, gender age, metabolic status, as well as methodological challenges [19-24] such as sample size, the number of miRNAs studied, blood collection practices and isolation methods [22]. Moreover, comorbidities such as obesity and diabetes can significantly affect plasma miRNA levels of putative cancer biomarkers $[25,26]$. Lastly, the miRNA detection method used, sample type tested (plasma versus serum), and the use of either spike-in or endogenous controls for normalization are the major determinants of study outcomes, regardless of the pathological condition in assessment [24].

Despite these challenges, this work on a purely Saudi, female patient cohort to investigate whether a stable circulating plasma miRNA signature could distinguish between disease-free individuals $(n=34)$ and early stages diagnoses of triple negative breast cancer (TNBC) $(n=36)$ and luminal breast cancer tumor $(n=57)$. Due to the relatively early diagnosis of cancer in this cohort, patient tissue biopsies were not available for analyses. Therefore, in order to compare differences in miRNA expression levels in tumor tissue versus plasma, plasma miRNA expression was compared to publically available RNASeq data from cancerous breast tissue and surrounding non-cancerous tissue available via The Cancer Genome Atlas (TCGA).

\section{Methods}

\section{Ethical statement}

Approval and written consent was obtained from all study participants for the use of their blood samples for research purposes. The study was approved by the Ethical Research Committee and Basic Research Committee on Clinical Research at KFSHRC, Riyadh, Saudi Arabia and was carried out under the terms of the Helsinki Declaration.

\section{Study cohort and clinical samples processing}

A total of 127 females, disease-free individuals $(n=34)$, triple-negative breast tumors (TNBC) $(n=36)$ and patients with luminal tumors $(n=57)$ were recruited for the study. All women were of Saudi background and recruited at the King Faisal Specialist Hospital (KFSHRC). Details of the study subjects with respect to the age of diagnosis, grade and lymph node status are reported in (Additional file 1: Table S1). All blood samples were obtained from patients before any cancer therapy was administered. Blood samples were collected by vene-section in EDTA blood collection tubes (BD Vactainer, Plymouth, UK) and kept at $4{ }^{\circ} \mathrm{C}$. The blood was then centrifuged within $2 \mathrm{~h}$ at $1500 \mathrm{~g}$ at $4^{\circ}$ $\mathrm{C}$ for $15 \mathrm{~min}$ to isolate the plasma. The plasma was collected and centrifuged again at $2500 \mathrm{~g}$ at $4{ }^{\circ} \mathrm{C}$ for $15 \mathrm{~min}$ (Heraeus multifug $3 \mathrm{~S}-\mathrm{R}-\mathrm{UK}$ ) to eliminate the debris. All samples were stored at $-80^{\circ} \mathrm{C}$.

\section{Isolation of microRNA from plasma}

RNA was isolated from the plasma using the miRNeasy Serum/Plasma Kit (Qiagen, Hilden, Germany), following the manufacturer's protocol with some modifications. RNA isolation was performed in duplicate. For the Qiagen kit assay, $1 \mathrm{ml}$ of QIAzol lysis reagent (Qiagen) was added to $200 \mu \mathrm{l}$ of plasma together with $1 \mu \mathrm{g}$ of carrier MS2 RNA (Roche). The samples were mixed and incubated for $5 \mathrm{~min}$ at room temperature. To monitor the RNA isolation before purification $3.5 \mu \mathrm{l}\left(1.6 \times 10^{8}\right.$ copies/ $\left.\mu \mathrm{l}\right)$ of C.elegansmiR-39 miRNA mimic spike-in control was added and $12 \mu \mathrm{g}$ of pure glycogen was added as a carrier/ co-precipitant. Next, $200 \mu \mathrm{l}$ of chloroform was added to the starting sample and mixed for $15 \mathrm{~s}$. Next, samples were incubated for $3 \mathrm{~min}$ at room temperature, and centrifuged for $15 \mathrm{~min}$ at $12,000 \mathrm{~g}$ at $4{ }^{\circ} \mathrm{C}$. $600 \mu \mathrm{l}$ of the aqueous phase was transferred to a new tube and $900 \mu \mathrm{l}$ of $100 \%$ ethanol was added to the spin column to allow all the RNA molecules to reach the binding condition. After mixing, the samples were transferred to RNeasy MinElute spin columns in a $2 \mathrm{ml}$ collection tube and spun for $15 \mathrm{~s}$ at $\geq 8000 \mathrm{~g}$. The columns were washed with two buffers from $700 \mu \mathrm{l}$ RWT buffer and then with $500 \mu \mathrm{l}$ RPE buffer (Qiagen, Hilden, Germany), with a short spin of $15 \mathrm{~s}$ at $\geq 8000$ g each time. Next, each column was washed with $500 \mu \mathrm{l}$ of $80 \%$ ethanol and centrifuged for $2 \mathrm{~min}$ at $\geq 8000$ g. Following $5 \mathrm{~min}$ high speed centrifugation, $14 \mu \mathrm{l}$ of RNase-free water could be used for elution. All RNA samples were frozen at $-80{ }^{\circ} \mathrm{C}$ until further analysis.

\section{Assessment of RNA quality and integrity}

Quality of RNA was assessed by Nanodrop ND-2000 (Wilmington, DE, USA). Chromatographic characteristics and integrity of all RNA samples were determined, included interpretation of the peak detection of different profiles, by means of RNA 6000 Nano LabChip, (Agilent Technologies, Waldbronn, Germany), Agilent 2100 Bioanalyzer system (Agilent Technologies, Santa Clara, USA) and the 2100 expert software tool (Agilent Technologies, Santa Clara, USA). 


\section{Quantitative real time polymerase chain reaction for mature miRNA expression profiling [13, 27-29]}

$250 \mathrm{ng}$ of the eluted RNA sample was used to make cDNA using a miScript RT II kit with miScript HiSpec buffer from Qiagen (Qiagen, Hilden, Germany). Briefly, the reaction was set up in the thermal cycler for $60 \mathrm{~min}$ at $37{ }^{\circ} \mathrm{C}$ followed by $5 \mathrm{~min}$ at $95{ }^{\circ} \mathrm{C}$. The cDNA was then diluted with $200 \mu \mathrm{l}$ of RNase-free water. For RT-qPCR a total of $2750 \mu$ l was prepared, made up of $(2 \mathrm{x}$ QuantiTect SYBR green PCR master mix, $10 \mathrm{x}$ miScript universal primers with a cDNA template and RNase free water). MicroRNAs screening was performed using miScript miRNA PCR Array Human Breast Cancer-MIHS-109Z (Qiagen, Hilden, Germany). The miRNA PCR Array Panel contains 84 mature miRNAs most relevant to breast cancer tumorigenesis. The final reaction volume was $25 \mu \mathrm{l}$ per well, enough to provide $1 \mathrm{ng}$ cDNA per well. The plates were run following a thermal cycling protocol: $95{ }^{\circ} \mathrm{C}$ for $15 \mathrm{~min}$ to activate the HotStar Taq DNA polymerase, 40 amplification cycles of $15 \mathrm{~s}$ at $94{ }^{\circ} \mathrm{C}, 30 \mathrm{~s}$ at $55^{\circ} \mathrm{C}, 30 \mathrm{~s}$ at $70{ }^{\circ} \mathrm{C}$ and at the end a melting curve program. All qPCR reactions were run in duplicate.

\section{Data processing and statistical analysis}

All samples passed 'Positive PCR Controls' (PPC) in which the acceptable range of $\mathrm{Ct}$ values was set to $19 \pm 2$. Both Reverse Transcription Controls (RTC) and (PPCs) were used to assess whether there had been any inhibition during the reverse transcription reaction. Avg $\mathrm{Ct}^{\text {miRTC }}-$ Avg $\mathrm{Ct}^{\mathrm{PPC}}$ was calculated for each sample (Avg "average"). A difference of greater than seven indicated impurities and reaction inhibition, cellular contamination was assessed using the mean $\mathrm{Ct}$ of the SNORNA (SNORD) controls. Only the non-zero values were considered. SNORD72 was excluded from computations as it performed poorly across samples. A sample with a mean $\mathrm{Ct}<32$ was taken to indicate cellular contamination. All samples used in the qRTPCR analysis were tested for quality and neither indication of cellular contamination nor reaction inhibition was detected. All samples collected were retained and none discarded. For miRNA to be within the detection limit, the $\mathrm{Ct}$ values were recommended to be between zero and 35 . Cel-correction to correct for technical variations that arise during extraction procedure, exogenous spike-in controls from C.elegans was used. The Qiagen miScript miRNA PCR Array Human Breast Cancer array panel contains two Cel-miR-39 spiked-in controls. The average $\mathrm{Ct}$ value recorded in each sample for the spiked-in controls from C.elegans, cel-39 was recorded and the median of average $\mathrm{Ct}$ values (of all samples) was found. The normalizing factor for each sample was calculated by subtracting the median of all average $\mathrm{Ct}^{\mathrm{cel}}$ values from the average $\mathrm{Ct}^{\mathrm{cel}}$ value for the sample. The ddCt computation $(\Delta \Delta \mathrm{Ct})$ for each of the three groups, the triple negative (TNBC) and the luminal tumors, average $\mathrm{dCt}(\Delta \mathrm{Ct})$ was calculated for each miRNA. The miRNAs with average $\mathrm{dCt}$ values $(\Delta \mathrm{Ct})$ below 15 and/or above 35 were excluded. ddCt $(\Delta \Delta \mathrm{Ct})$ of a given miRNA for a given pair of groups was computed by finding the difference between the average $\mathrm{dCt}(\Delta \mathrm{Ct})$ of the respective groups; for example, $\Delta \Delta \mathrm{Ct}(\mathrm{ddCt}$ (Triple Negative vs. Controls $))=[$ Average $\mathrm{dCt}(\mathrm{TNBC})]-[$ Average $\mathrm{dCt}$ (Controls)]. The relative expression of a given miRNA between any two groups was assessed by computing $2^{(-\mathrm{ddCt})}$ $\left(2^{(-\Delta \Delta \mathrm{Ct})}\right)$. A differential set was identified using the ddCt $(\Delta \Delta \mathrm{Ct})$ method proposed by Livak et al. [30]. The MannWhitney unpaired test and Benjamin-Hochberg multiple testing corrections were used to determine significant differences in miRNA expression levels between groups [31]. All qPCR reactions were run in duplicate.

\section{Bioinformatics analysis}

The miRNA targets and the biological pathways they were involved in were predicted using the microT-CDS algorithm and mirPath v.2.0 available on the web-based server DIANA. The micro-T threshold for target prediction was set at 0.8 and targeted pathways were considered significant at a $p$-value $<0.05[32,33]$. Hierarchical clustering was performed using GeneSpring GX 14.5.

\section{Receiver operating characteristic (ROC) curves}

Receiver Operating Characteristic (ROC) curves were generated using the web-based tool ROCCET [34] for finding two sets of miRNAs that could best differentiate (i) triplenegative tumour samples from normal (control) samples and (ii) luminal tumour samples from normal (control) samples. ROC (Receiver Operating Characteristic) curves were then generated by Monte-Carlo Cross Validation (MCCV). The procedure was performed repeatedly to calculate the performance and confidence interval of each model.

\section{Comparison of miRNA levels in tissue and plasma}

Since plasma levels of miRNA are not necessarily a reflection of tissue levels [35-38] and tumor tissue was not available from patients recruited for this study as plasma was collected pre-cancer therapy, publicly available data from the TCGA (The Cancer Genome Atlas) [39] was used to determine whether circulating plasma levels were distinct from breast cancer tissue and surrounding non-cancerous tissue levels. We compared the observed miRNA plasma levels with the tissue levels of corresponding miRNA precursors from the TCGA study. The tissue level expression of miRNA precursors, available as RPKM (reads per kilobase of transcript per million) values was obtained for control samples $(n=$ $87)$, luminal samples $(n=120)$ and Triple Negative samples $(n=38)$ from TCGA. The RPKM values of the precursors in tissue and the expression value for miRNAs 
in the current study were $\log _{2}$ transformed and autoscaled, to ensure the datasets are comparable.

Auto scaled value $=(x-\mu) / \delta$

The tissue RPKM and the plasma cel-corrected $C_{t}$ values are normalized to the mean $(\mu)$ and standard deviation $(\delta)$ for each of the data. Since a miRNA precursor can give rise to an active form from each arm, we compared both the $3^{\prime}$ and $5^{\prime}$ active forms were matched to the same precursor. The 18 differentially expressed active miRNA forms mapped to 17 precursor miRNAs from TCGA. Then we analyzed the expression trends of a given miRNA across tissue and plasma samples.

\section{Results}

Differences in circulating miRNAs between breast cancer patients and normal samples

Comparative analysis identified an initial set of 18 circulating miRNAs (Table 1), which because of their differential presence between the patient groups and healthy controls, were further examined by cancer type. Figure 1 illustrates relative expression of these 18 miRNAs subdivided into three groups: TNBC plasma vs. disease free plasma (Group A; $n=8$ miRNAs: hsa-miR-29c-3p, hsa-miR-195-5p, hsamiR-210-3p, hsa-miR-19b-3p, hsa-miR-19a-3p, hsa-miR22-3p, hsa-miR-7-5p, hsa-miR-15a-5p); luminal patient plasma vs. disease free plasma (Group B; $n=5$ miRNAs: hsa-let-7c-5p, hsa-miR-489-3p, hsa-miR-340-5p, hsa-miR199a-5p, hsa-miR-328-3p); lastly, all breast cancer patients (irrespective of subtype) vs. disease free plasma (Group C; $\mathrm{n}=5$ miRNAs: hsa-let-7i-5p, hsa-miR-25-3p, hsa-miR-165p, hsa-let-7b-5p, hsa-miR-199a-3p).

\section{Comparison of circulating miRNAs levels with cancerous and non-cancerous tissue expression}

Next, the tissue expression trends of the corresponding 17 miRNA precursors in relation to their active forms in plasma were measured (Fig. 2). Since plasma collected from all patients enrolled in this study occurred prior to chemotherapy administration and/or tumor biopsy, miRNA breast cancer tissue and non-cancerous tissue expression values

Table 1 Fold change (FC) and p-values of the 18 significant miRNA

\begin{tabular}{|c|c|c|c|c|c|c|c|c|}
\hline \multirow[b]{2}{*}{ miRNA ID } & \multicolumn{4}{|c|}{ Triple Negative } & \multicolumn{4}{|c|}{ Luminal } \\
\hline & $\begin{array}{l}2 \wedge-(T N B C- \\
C)^{A}\end{array}$ & $\mathrm{P}_{\mathrm{P}}$ (Corr) TNBC vs & $\begin{array}{l}\text { Regulation (TNBC vs } \\
\text { C) }\end{array}$ & $\begin{array}{l}\text { FC (TNBC vs } \\
C)^{F}\end{array}$ & $\begin{array}{l}2^{\wedge}-(L- \\
C)^{B}\end{array}$ & $p_{p}$ (Corr) L vs C & $\begin{array}{l}\text { Regulation (L vs } \\
\text { C) }\end{array}$ & $\begin{array}{l}F C(L \vee v S \\
C)^{F}\end{array}$ \\
\hline hsa-let-7b-5p & 2.14 & 0.006 & Up & 2.14 & 2.29 & 0.000 & Up & 2.29 \\
\hline hsa-let-7c-5p & 1.72 & 0.031 & Up & 1.72 & 1.95 & 0.001 & Up & 1.95 \\
\hline hsa-let-7i-5p & 1.71 & 0.008 & Up & 1.71 & 1.60 & 0.008 & Up & 1.60 \\
\hline hsa-miR-15a-5p & 2.01 & 0.006 & Up & 2.01 & 0.91 & 0.977 & Up & -1.09 \\
\hline hsa-miR-16-5p & 2.20 & 0.000 & Up & 2.20 & 1.68 & 0.008 & Up & 1.68 \\
\hline hsa-miR-195-5p & 1.84 & 0.004 & Up & 1.84 & 1.60 & 0.011 & Up & 1.60 \\
\hline $\begin{array}{l}\text { hsa-miR-199a- } \\
3 p\end{array}$ & 0.36 & 0.002 & Down & -2.81 & 0.36 & 0.003 & Down & -2.80 \\
\hline $\begin{array}{l}\text { hsa-miR-199a- } \\
5 p\end{array}$ & 0.46 & 0.021 & Down & -2.19 & 0.25 & 0.001 & Down & -3.94 \\
\hline hsa-miR-19a-3p & 1.81 & 0.006 & Up & 1.81 & 1.29 & 0.205 & Up & 1.29 \\
\hline hsa-miR-19b-3p & 1.86 & 0.006 & Up & 1.86 & 1.27 & 0.203 & Up & 1.27 \\
\hline hsa-miR-210-3p & 1.91 & 0.000 & Up & 1.91 & 1.23 & 0.646 & Up & 1.23 \\
\hline hsa-miR-22-3p & 1.93 & 0.006 & Up & 1.93 & 1.08 & 0.083 & Up & 1.08 \\
\hline hsa-miR-25-3p & 2.02 & 0.001 & Up & 2.02 & 2.01 & 0.001 & Up & 2.01 \\
\hline hsa-miR-29c-3p & 1.66 & 1.010 & Up & 1.66 & 1.19 & 0.203 & Up & 1.19 \\
\hline hsa-miR-328-3p & 1.58 & 0.563 & Up & 1.58 & 20.96 & 0.000 & Up & 20.96 \\
\hline hsa-miR-340-5p & 0.65 & 0.043 & Down & -1.54 & 0.55 & 0.008 & Down & -1.81 \\
\hline hsa-miR-489-3p & 1.85 & 0.057 & Up & 1.85 & 2.26 & 0.000 & Up & 2.26 \\
\hline hsa-miR-7-5p & 2.30 & 0.003 & Up & 2.30 & 2.01 & 0.012 & Up & 2.01 \\
\hline
\end{tabular}

TNBC Triple Negative Breast Cancer

$C$ - Disease-free individuals used as Controls

L - Luminal Breast Cancer

A : $2^{\wedge}-\left(\right.$ TNBC-N) represents $2^{\wedge} \mathrm{ddCt}$ values of Triple Negative patient's samples as compared to those of disease-free individuals

$P:$ Corrected $p$-values $\leq 0.01$ for a given pair of conditions are shown in bold

$F$ : Fold change values $\geq 1.5$ are shown in bold

${ }^{B}: 2^{\wedge}-(\mathrm{L}-\mathrm{N})$ represents $2^{\wedge} \mathrm{ddCt}$ values of Luminal patients' samples as compared to those disease-free individuals 


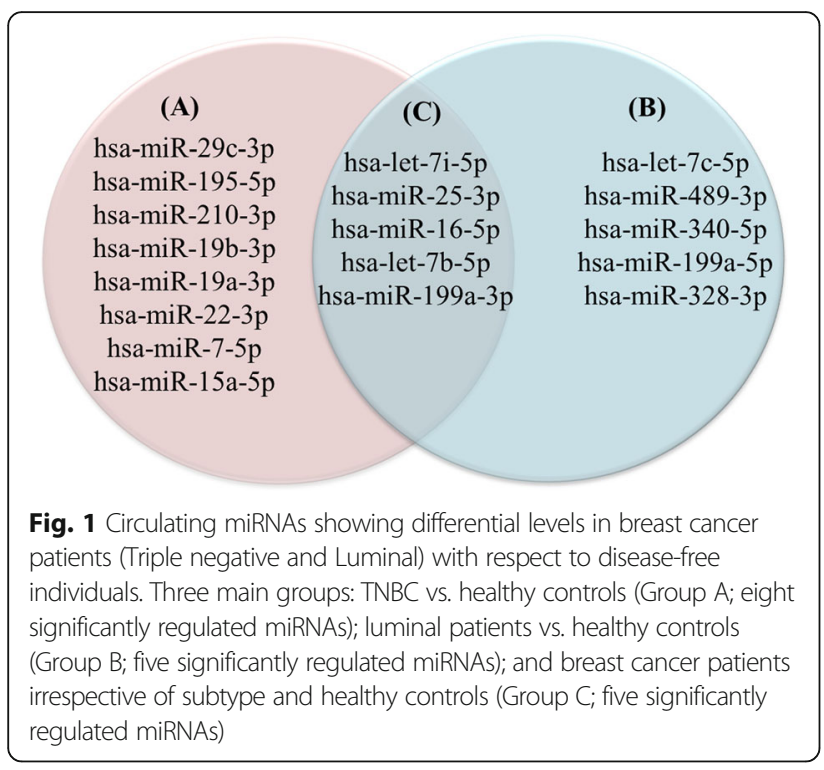

were obtained from publically available TCGA RNASeq data; non-cancerous tissue samples $(n=87)$, luminal samples $(n=120)$ and Triple Negative samples $(n=38)$. Expression trends for some miRNAs (hsa-miR-19a, hsa-miR-19b, hsa-miR-210, hsa-miR-16, hsa-miR-7 and to a certain extent hsa-miR-15a) were similar in both tissue and plasma. Tissue and plasma levels of non-diseased controls compared against both cancer patient groups showed a broad reversal of the trend. For example, tumor repressor miRs hsa-let-7c and hsa-miR-195 were significantly decreased in both luminal and TNBC breast cancer tissue levels (TCGA) and increased in non-tumor tissue samples. Slight variations of this pattern were observed for hsa-miR-489, hsamiR-328, hsa-miR-25, hsa-let-7i, hsa-let-7b, hsa-miR-29c, hsa-miR-199a, hsa-miR-340 and hsa-miR-22.

\section{Pathway analysis of target genes}

Lastly, a pathway analyses was performed on the target genes of the 18 differential miRNAs identified in this study (Additional file 1: Figure S1). Not surprisingly, many of them were involved in signaling functions, namely the PI3K-Akt, mTOR, p53, TGF-beta, Wnt, FoxO, estrogen signaling and Hippo signaling pathways. However, the most significantly enriched pathways were the ECMreceptor interaction (Extracellular Matrix) and the fatty acid biosynthesis (FASN) pathways. Gene targets of the let-7 family were found to be involved in ECM receptor interaction while the fatty acid biosynthesis pathway (FASN) was shown to be enriched mainly by hsa-miR-165p, hsa-miR-15a-5p and hsa-miR-195-5p. Both hsa-miR15a-5p and hsa-miR-195-5p were enriched exclusively in the plasma of TNBC patients (see Fig. 1).

\section{Receiver operating characteristic (ROC) curves}

In order to assess the potential of each of these miRNAs as cancer biomarkers, we generated ROC curves. Since Univariate AUC ROC curves looked promising, whether a more robust prediction could be made using multiple miRNAs was explored. A panel of seven miRNAs consisting of

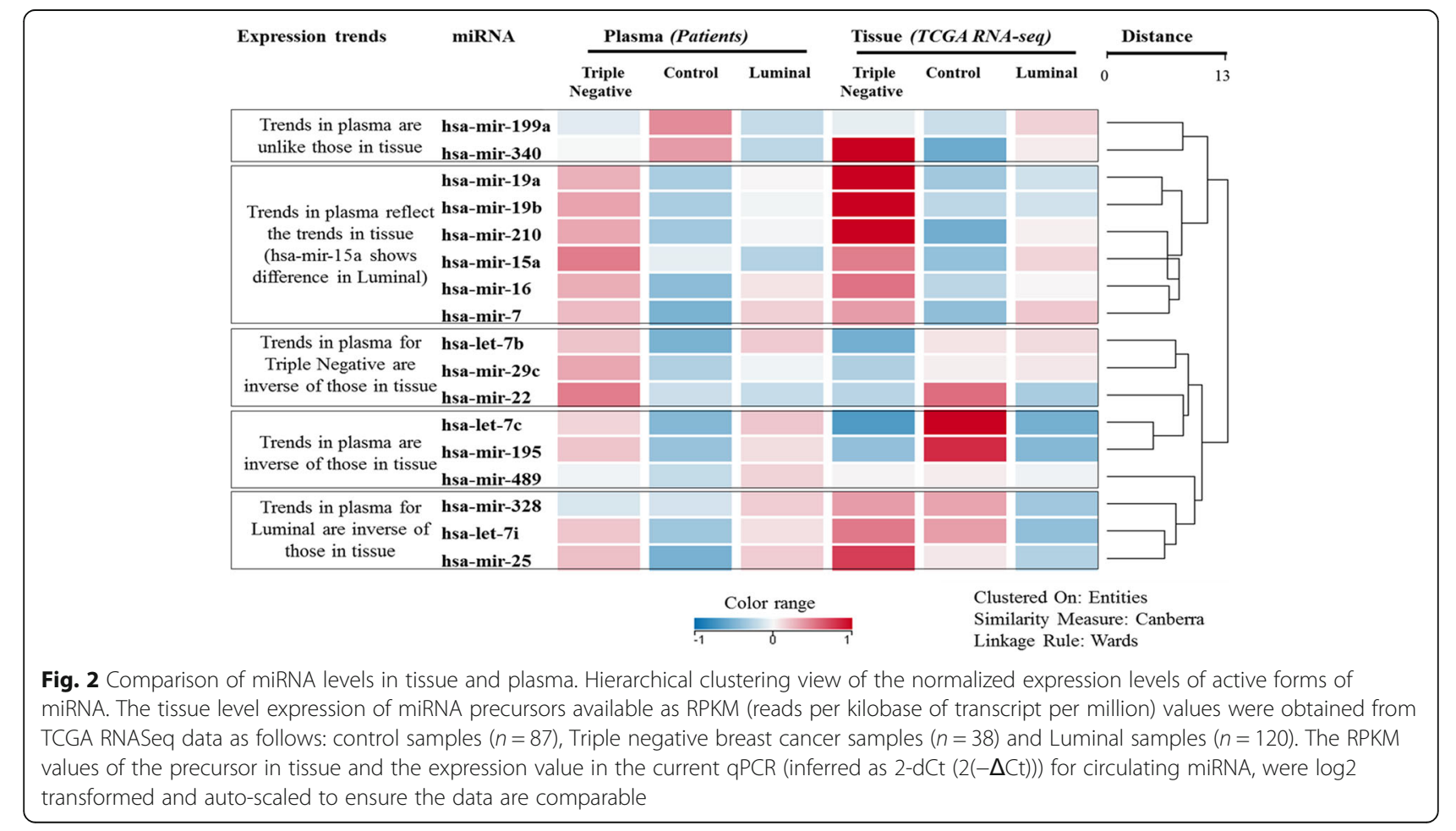


hsa-miR-199a-3p, hsa-miR-15a-5p, hsa-let-7c-5p, hsa-miR7-5p, hsa-miR-195-5p, hsa-miR-489-3p and hsa-let-7i-5p showed the maximum discriminatory potential between TNBC patient plasma and disease-free plasma (Fig. 3a). Similarly, a panel of five miRNAs consisting of hsa-miR328-3p, hsa-miR-199a-3p, hsa-let-7i-5p, hsa-miR-195-5p and hsa-miR-25-3p best predicted luminal tumor patients from disease-free individuals (Fig. 3b). The Univariate AUC statistic for each miRNA is provided (Additional file 1: Table S2).

\section{Discussion}

In less developed countries, including those of the Middle East, breast cancer accounted in 2012 for $25 \%$ of all reported cancer cases in females. It has been estimated that in 2020 more than 1.9 million women will be diagnosed with breast cancer, marking an increase of $18.4 \%$ [40]. Given the expected increase in female breast cancer diagnosis, the aim of this study was to discover whether any putative circulating miRNA biomarkers, could be differentially detected in the plasma of early stage, treatmentnaïve female breast cancer patients. Analyses were performed on plasma isolated from healthy, cancer-free females $(n=34)$, cancer therapy naïve patients diagnosed with triple-negative breast cancer tumors (TNBC) $(n=36)$ and finally, cancer therapy naïve patients with luminal tumors $(n=57)$. As patient tumor biopsies were not available at the time of plasma collection, plasma miRNA expression levels in cancer groups were analyzed not only against the plasma of healthy but also against publically available RNASeq data from non-cancerous tissue samples $(n=87)$, luminal samples $(n=120)$ and triple negative samples $(n=$ 38), provided by the (The Cancer Genome Atlas (TCGA)).

It is exceptionally challenging to discuss the results of miRNA biomarker studies in the context of the literature as reports are very contradictory. A meta-analyses performed by Leidner et al. [41] demonstrated major inconsistencies in $\mathrm{qPCR}$ as well as genome-wide approaches for detecting miR biomarkers. For example, with the exception of miR155 and miR-21, none of the 25 miRNAs analyzed by qPCR by eight independent groups; whose cohort sizes were similar to the one used in this study, were detected to be in agreement by more than one study. Furthermore, the findings of significantly elevated circulating miR-155 and miR21 by qPCR in breast cancer were actually contradicted by subsequent data reported by genome-wide approaches leading to what Leidner refers to as a dampening of enthusiasm for miRNA biomarkers [41]. However, the relatively pure genetic background of the patient population may increase the likelihood of reproducibility as well as the possibility for clinical biomarker application.

As described by Witwer et al. [19] and Chen et al. [42], the composition of circulating miRNAs in cancer patients is governed by the following: 1) active secretion and/or passive leakage of miRNA from tumor cells, 2) increased cellular production and secretion, 3) enhanced selective secretion, and 4) changes in miRNA stability. Similarly, down-regulation of miRNA in the plasma may indicate reduced secretion, increased retention and/or possibly represent a general neoplastic state [19]. For these reasons as well as the fact that biopsies from chemotherapy naïve patient were not available, the differentially regulated miRNAs
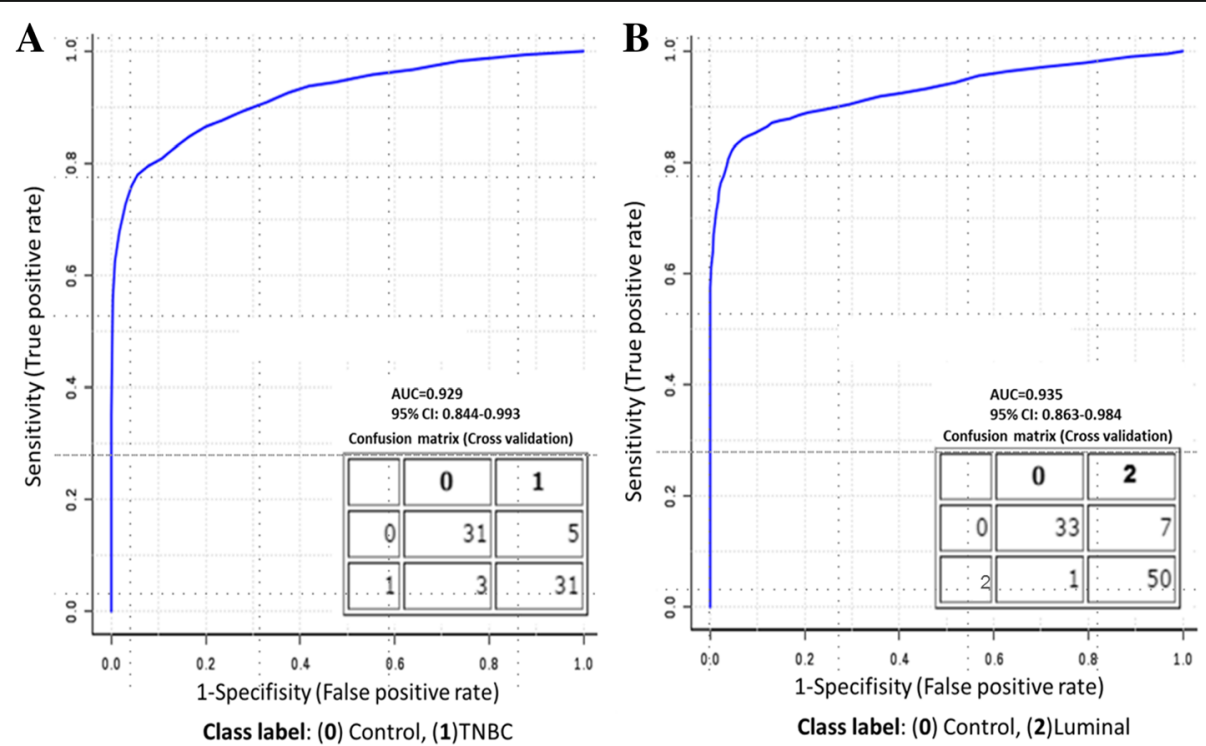

Fig. 3 Receiver-operating characteristic (ROC) curve analyses (a) Panel of 7 miRNAs consisting of hsa-miR-199a-3p, hsa-miR-15a-5p, hsa-let-7c-5p, hsa-miR-7-5p, hsa-miR-195-5p, hsa-miR-489-3p and hsa-let-7i-5p showed the maximum discriminatory potential between triple negative tumors and disease-free individuals. Similarly, a five miRNA (b) Panel consisting of hsa-miR-328-3p, hsa-miR-199a-3p, hsa-let-7i-5p, hsa-miR-195-5p and hsa-miR-25-3p best differentiated luminal tumors patients from the disease-free individuals 
identified in the plasma samples in this study were compared with tissue levels of miRNA precursors from The Cancer Genome Atlas (TCGA) (Fig. 2).

This analysis performed on this patient cohort led to the identification of three broad categories representing distinct expression patterns of miRNAs. The first category consists of hsa-miR-19a, hsa-miR-19b, hsa-miR-210, hsamiR-15a, hsa-miR-16 and hsa-miR-7 which are overexpressed in TNBC tissues as well as plasma. Therefore, these miRNAs may directly reflect TNBC tumor biology. To date, many studies have confirmed hsa-miR-19a/b oncogenic role in TNBC tumor biology by repressing PTEN and activating NF-kB [43] and levels of circulating miR-19 correlated with response to neoadjuvant epirubicin + paclitaxel chemotherapy regimen in Stage II and III patients with luminal A breast tumors [44]. In a Japanese TNBC patient cohort, high hsa-miR-210 expression was identified as an independent factor indicating poor prognosis for TNBC [45]. In contrast, members of the miR-15 family have tumour suppressor properties. Hsa-miR-16-5p and hsa-miR-15a-5p are involved in the cell cycle, differentiation, proliferation, hormone regulation and immune response [46]. Various studies reported their downregulation in most tumours [47]. However, miR-15 family miRNAs are strongly regulated by hormones [48]. Given their multiple functions and complicated regulation, it is unlikely that miR-15 family members would make an sufficient early biomarker for breast cancer. The second category of miRNAs consisted of hsa-miR-199a and hsamiR-340 which were differentially regulated in tissue and plasma. In contrast to the first group, these miRNAs were decreased in breast cancer patient plasma compared to healthy controls. The third category has several distinct sub-groups. An inverse pattern between plasma and tissue levels specific to TNBC patients was observed for hsa-let7b, hsa-miR-29c, and hsa-miR-22. In all cases the plasma miRNAs levels were higher than the tissue levels, supporting evidence of cancer cell secretion of miRNAs.

The main finding of this study is that blood based screening of has-miR-195 and let-7 may help to identify and diagnose early stages of breast cancer patients. Elevated circulating levels of let-7 family members (has-let$7 \mathrm{~b}$, has-let-7c and has-let-7i) were observed. Studies using breast cancer cell lines [36] and other cancer cell lines [37] have reported the selective release of tumor suppressor miRNAs into extra-cellular fluids. Therefore, extra-cellular miRNAs are not merely the artefacts excreted by dead tumour cells but key players assisting in tumor development and metastasis by promoting cancer-host cross talk [49]. As illustrated by Falcone et al. [50], tumor cells use a multi-pronged approach to create a metastatic niche by selectively secreting out tumor suppressor miRNAs, thereby overcoming immune surveillance by repressing the immune system and promoting angiogenesis. Various members of the let-7 family have been reported to be down-regulated in cancer tissues. Furthermore, it has been shown that the restoration of let-7 levels in cells could be an effective cancer therapy [51]. Thus it is probable that breast cancer tumour cells selectively secrete tumor suppressor miRNAs to maintain oncogenesis as suggested by Ohshima et al. [35]. Let-7 was also increased in the TNBC patient plasma from an Irish patient cohort [52] while in this population, let-7 was only increased in luminal patients. In contrast, an Indian cohort [53] observed decreased miR-195, and increased Let-7 miRNA in circulating plasma of TNBC patients. These highly variable results may be due to a variety of patient variables such as metabolic status, age, cancer stage; controls used, and may also highlight the influence of genetic background on miRNA expression. At the time of this manuscript revision, patient recruitment is currently ongoing for a robust blinded validation experiment.

Based on the pathway analysis performed in DIANA [32, 33], the 18 miRNAs detected in this study and their targets are extensively involved in FASN pathways, ECM-receptor interaction, PI3K-Akt, mTOR, p53, TGFbeta, Wnt, FoxO, estrogen signaling and Hippo signaling pathways, all critical for carcinogenesis. Chen et al. [54] reported hsa-miR-195-5p as a direct regulator of GLUT3 and the increased amounts of GLUT3 transcripts seem to facilitate accelerated metabolism, high glucose requirements, and increased glucose uptake in malignant cells. Using cell lines, Singh et al. [55] demonstrated the anti-cancer activity of hsa-miR-195 and suggested overexpression of hsa-miR-195 as a potential therapy for breast cancer. In this study, circulating hsa-mir-195 levels in TNBC plasma are higher than those in healthy individuals. Likewise, an increased systemic miR-195 levels was observed in blood of breast cancer patients [52, 56-58]. Non-cancerous TCGA tissue had high levels of tumor suppressor hsa-miR-195 while TNBC cancer tissue had low levels suggesting that hsa-miR-195 is secreted out of cancer cells, possibly to facilitate increased GLUT3 expression.

Finally, in the panel of miRNAs selected for distinguishing both TNBC and luminal patients from healthy controls, the receiver operating characteristic (ROC) analysis consistently included hsa-let-7 and hsa-miR-195. Compared to cancer-free plasma samples, let-7 miRNA was most elevated and associated with luminal breast cancer diagnosis, irrespective of subtype, and miR-195 was elevated in TNBC plasma and most associated with TNBC breast cancer patients. In contrast, hsa-miR-195 and let-7 miRNAs were absent from cancerous TCGA tissue and strongly expressed in surrounding non-tumor tissue indicating that breast cancer tumor cells may selectively export hsa-miR-195 and let-7 miRNAs. Taken together, these observations suggest that any study evaluating the use of 
the over-expressed hsa-let-7 family and/or hsa-miR-195 as anti-cancer therapy should consider that tumor cell machinery may actively target and excrete hsa-miR-195, thereby neutralizing its anti-cancer effect $[59,60]$. However, these miRNAs may be of potential use in the development of a blood based screening test to complement and improve early detection of breast cancer [58].

\section{Conclusion}

Plasma sampling from patients remains the least invasive method for identifying biomarkers so any circulating miRNA with disease specific expression would be advantageous to clinicians. Breast cancer specific expression requires that the putative biomarker expression remains tightly linked to biological changes occurring during the onset of tumor growth and through metastasis. Results from this study suggest that both miR-195 and let-7 make satisfactory candidates for biomarkers. However, since levels of let-7 have also been reported to be increased in the serum/plasma of patients with other types of cancer, a biomarker test alone would not be sufficient to determine a diagnosis. However, let-7 may be a better candidate than other miRs such as miR-15 or miR-29 which are regulated not only by the process of tumorgenesis but also by hormones, which may lead to more variability in results. Furthermore, while some studies have indicated that the restoration of let- 7 and miR-195 may be a potential therapy for cancer; this study found that circulating hsa-miR-195 levels in TNBC plasma are already significantly higher than those of healthy individuals. These results also suggest that tumor cells may selectively export hsa-miR-195 and let-7 miRNAs thereby neutralizing their potential therapeutic effect $[59,60]$. Finally, the model constructed by ROC of a panel of seven miRNAs showed the maximum discriminatory potential between TNBC patient plasma and disease-free plasma while a panel of five miRNAs best predicted luminal tumor patients from disease-free individuals. Future experiments performed on this patient cohort should confirm findings in plasma, patient tissue, and track these markers through the course of treatment (including tissue from mastectomies) and during remission. While large scale studies are necessary to confirm these results before they can be applied into clinical practice, the miRNAs differentially detected in the plasma of breast cancer patients in this study warrant further investigation.

\section{Additional files}

Additional file 1: Table S1. Characteristics of breast cancer patients. Figure S1. Heat map of pathways enriched by the target genes for the 18 differential miRNAs. The signalling pathways, namely PI3K-Akt, mTOR, p53, TGF-beta, Wnt, FoxO, estrogen, Hippo signalling and ECM receptor interaction, fatty acid metabolism and fatty acids biosynthesis pathways are enriched. Table S2. Univariate AUC statistics for the differential miRNAs based on ROC Analysis. (DOCX $1154 \mathrm{~kb}$ )

\section{Abbreviations \\ Ct : Threshold cycle; miRNA: microRNA; qRT-PCR: Quantitative Real Time Polymerase Chain Reaction; RNASeq: Ribonucleic acid sequencing; ROC: Receiver Operating Characteristic; RPKM: Reads Per Kilobase of transcript per Million mapped reads}

\section{Acknowledgments}

The authors would like to thank all the patients and volunteers at KFSHRC who participated in the study. In addition, the authors would like to thank the RC-Logistics Management Office (RCLMO) at KFSHRC in Riyadh, SA.

Funding

King Faisal Specialist Hospital and Research Centre [RAC\#2160029 and 2110016]. Funding for open access charge: King Faisal Specialist Hospital and Research Centre. L'Oréal-UNESCO (FWIS) Award for Dr.Amal Qattan,PhD Middle East Fellowship 2017.

Availability of data and materials

The datasets used during the current study are available from the corresponding author on reasonable request.

\section{Authors' contributions \\ AQ (conceived the study, analysis, interpretation of data and writing the manuscript).AQ and $\mathrm{HI}$ (carried out the experimental work and data extraction).CE (sample collection, processing, storage and information management). TT, and WA (acquisition of clinical data and revising critically the manuscript). AQ and SA (critically reviewed and revised the manuscript). All authors read and approved the final manuscript and agreed to be accountable for all aspects of the work.}

\section{Authors' information}

All authors from King Faisal Specialist Hospital and Research centre, Riyadh, Saudi Arabia

\section{Ethics approval and consent to participate}

Approval and written consent was obtained from all study participants for the use of their blood samples for research purposes. The study was approved by the Ethical Research Committee and Basic Research Committee (Approval number: RAC\#2160029 and 2,110,016) on Clinical Research at KFSHRC, Riyadh, Saudi Arabia and was carried out under the terms of the Helsinki Declaration.

\section{Consent for publication}

NA

\section{Competing interests}

The authors declare that they have no competing interests.

\section{Publisher's Note}

Springer Nature remains neutral with regard to jurisdictional claims in published maps and institutional affiliations.

\footnotetext{
Author details

${ }^{1}$ Breast Cancer Research, Department of Molecular Oncology, King Faisal Specialist Hospital and Research Centre, P.O.Box 3354, Riyadh 11211, Saudi Arabia. ${ }^{2}$ Department of Biochemistry and Molecular Medicine, School of Medicine and Health Sciences (SMHS), George Washington University, 2600 Virginia Avenue, NW, Suite 300, Washington, DC 20037, USA. ${ }^{3}$ College of Medicine, Alfaisal University, P.O.Box 50927, Riyadh 11533, Saudi Arabia. ${ }^{4}$ College of Medicine, Princess Nourah Bint Abdulrahman University, Riyadh, Saudi Arabia. ${ }^{5}$ Department of Surgery, King Faisal Specialist Hospital and Research centre, Riyadh, Saudi Arabia. ${ }^{6}$ Department of Oncology, King Faisal Specialist Hospital and Research centre, Riyadh, Saudi Arabia.
} 
Received: 27 February 2017 Accepted: 13 November 2017 Published online: 28 November 2017

\section{References}

1. Coughlin SS, Ekwueme DU. Breast cancer as a global health concern. Cancer Epidemiol. 2009;33(5):315-8

2. Siegel RL, Miller KD, Jemal A. Cancer statistics, 2017. CA Cancer J Clin. 2017; 67(1):7-30.

3. Weinberger M, Saunders AF, Samsa GP, Bearon LB, Gold DT, Brown JT, Booher P, Loehrer PJ. Breast cancer screening in older women: practices and barriers reported by primary care physicians. J Am Geriatr Soc. 1991; 39(1):22-9.

4. Graham LJ, Shupe MP, Schneble EJ, Flynt FL, Clemenshaw MN, Kirkpatrick AD, Gallagher C, Nissan A, Henry L, Stojadinovic A, et al. Current approaches and challenges in monitoring treatment responses in breast cancer. J Cancer. 2014;5(1):58-68.

5. Asaga S, Kuo C, Nguyen T, Terpenning M, Giuliano AE, Hoon DS. Direct serum assay for microRNA-21 concentrations in early and advanced breast cancer. Clin Chem. 2011:57(1):84-91.

6. Chan M, Liaw CS, Ji SM, Tan HH, Wong CY, Thike AA, Tan PH, Ho GH, Lee AS. Identification of circulating microRNA signatures for breast cancer detection. Clin Cancer Res. 2013;19(16):4477-87.

7. Farazi TA, Horlings HM, Ten Hoeve JJ, Mihailovic A, Halfwerk H, Morozov P, Brown M, Hafner M, Reyal F, van Kouwenhove M, et al. MicroRNA sequence and expression analysis in breast tumors by deep sequencing. Cancer Res. 2011:71(13):4443-53.

8. Roth C, Rack B, Muller V, Janni W, Pantel K, Schwarzenbach H. Circulating microRNAs as blood-based markers for patients with primary and metastatic breast cancer. Breast Cancer Res. 2010;12(6):R90.

9. Zhu W, Qin W, Atasoy U, Sauter ER. Circulating microRNAs in breast cancer and healthy subjects. BMC Res Notes. 2009;2:89.

10. Ambros V. The functions of animal microRNAs. Nature. 2004:431(7006):350-5.

11. Bartel DP. MicroRNAs: genomics, biogenesis, mechanism, and function. Cell. 2004;116(2):281-97.

12. Bartel DP. MicroRNAs: target recognition and regulatory functions. Cell. 2009;136(2):215-33.

13. Mitchell PS, Parkin RK, Kroh EM, Fritz BR, Wyman SK, Pogosova-Agadjanyan EL, Peterson A, Noteboom J, O'Briant KC, Allen A, et al. Circulating microRNAs as stable blood-based markers for cancer detection. Proc Natl Acad Sci U S A. 2008;105(30):10513-8.

14. Gilad S, Meiri E, Yogev Y, Benjamin S, Lebanony D, Yerushalmi N, Benjamin $\mathrm{H}$, Kushnir M, Cholakh H, Melamed N, et al. Serum microRNAs are promising novel biomarkers. PLoS One. 2008;3(9):e3148.

15. Davis-Dusenbery BN, Hata A. MicroRNA in cancer: the involvement of aberrant MicroRNA biogenesis regulatory pathways. Genes Cancer. 2010; 1(11):1100-14

16. Jansson MD, Lund AH. MicroRNA and cancer. Mol Oncol. 2012;6(6):590-610.

17. Moussay E, Wang K, Cho JH, van Moer K, Pierson S, Paggetti J, Nazarov PV, Palissot V, Hood LE, Berchem G, et al. MicroRNA as biomarkers and regulators in B-cell chronic lymphocytic leukemia. Proc Natl Acad Sci U S A. 2011;108(16):6573-8

18. Zhang J, Zhao H, Gao Y, Zhang W. Secretory miRNAs as novel cancer biomarkers. Biochim Biophys Acta. 2012;1826(1):32-43.

19. Witwer KW. Circulating microRNA biomarker studies: pitfalls and potential solutions. Clin Chem. 2015;61(1):56-63.

20. Farina NH, Wood ME, Perrapato SD, Francklyn CS, Stein GS, Stein JL, Lian JB. Standardizing analysis of circulating microRNA: clinical and biological relevance. J Cell Biochem. 2014;115(5):805-11.

21. McDonald JS, Milosevic D, Reddi HV, Grebe SK, Algeciras-Schimnich A. Analysis of circulating microRNA: preanalytical and analytical challenges. Clin Chem. 2011;57(6):833-40.

22. Moldovan L, Batte KE, Trgovcich J, Wisler J, Marsh CB, Piper M. Methodological challenges in utilizing miRNAs as circulating biomarkers. J Cell Mol Med. 2014;18(3):371-90.

23. Pritchard CC, Kroh E, Wood B, Arroyo JD, Dougherty KJ, Miyaji MM, Tait JF, Tewari M. Blood cell origin of circulating microRNAs: a cautionary note for cancer biomarker studies. Cancer Prev Res. 2012;5(3):492-7.

24. Marabita F, de Candia P, Torri A, Tegner J, Abrignani S, Rossi RL. Normalization of circulating microRNA expression data obtained by quantitative real-time RT-PCR. Brief Bioinform. 2016;17(2):204-12.
25. Zampetaki A, Kiechl S, Drozdov I, Willeit P, Mayr U, Prokopi M, Mayr A Weger $\mathrm{S}$, Oberhollenzer F, Bonora $\mathrm{E}$, et al. Plasma microRNA profiling reveals loss of endothelial miR-126 and other microRNAs in type 2 diabetes. Circ Res. 2010;107(6):810-7.

26. Pescador N, Perez-Barba M, Ibarra JM, Corbaton A, Martinez-Larrad MT, Serrano-Rios M. Serum circulating microRNA profiling for identification of potential type 2 diabetes and obesity biomarkers. PLoS One. 2013;8(10): e77251.

27. McAlexander MA, Phillips MJ, Witwer KW. Comparison of methods for miRNA extraction from plasma and quantitative recovery of RNA from cerebrospinal fluid. Front Genet. 2013;4:83.

28. Hatse S, Brouwers B, Dalmasso B, Laenen A, Kenis C, Schoffski P, Wildiers H. Circulating MicroRNAs as easy-to-measure aging biomarkers in older breast cancer patients: correlation with chronological age but not with fitness/ frailty status. PLoS One. 2014:9(10):e110644.

29. Moret I, Sanchez-Izquierdo D, Iborra M, Tortosa L, Navarro-Puche A, Nos P, Cervera J, Beltran B. Assessing an improved protocol for plasma microRNA extraction. PLoS One. 2013;8(12):e82753.

30. Livak KJ, Schmittgen TD. Analysis of relative gene expression data using real-time quantitative PCR and the $2(-$ Delta Delta $C(T))$ method. Methods. 2001:25(4):402-8.

31. Matamala N, Vargas MT, Gonzalez-Campora R, Minambres R, Arias JI, Menendez P, Andres-Leon E, Gomez-Lopez G, Yanowsky K, CalveteCandenas J, et al. Tumor microRNA expression profiling identifies circulating microRNAs for early breast cancer detection. Clin Chem. 2015;61(8):1098-106.

32. Papadopoulos GL, Alexiou P, Maragkakis M, Reczko M, Hatzigeorgiou AG. DIANA-mirPath: integrating human and mouse microRNAs in pathways. Bioinformatics. 2009:25(15):1991-3.

33. Maragkakis $M$, Reczko M, Simossis VA, Alexiou P, Papadopoulos GL, Dalamagas T, Giannopoulos G, Goumas G, Koukis E, Kourtis K, et al. DIANAmicroT web server: elucidating microRNA functions through target prediction. Nucleic Acids Res. 2009;37(Web Server issue):W273-6.

34. Xia J, Broadhurst DI, Wilson M, Wishart DS. Translational biomarker discovery in clinical metabolomics: an introductory tutorial. Metabolomics. 2013;9(2): 280-99.

35. Ohshima K, Inoue K, Fujiwara A, Hatakeyama K, Kanto K, Watanabe $Y$, Muramatsu K, Fukuda Y, Ogura S, Yamaguchi K, et al. Let-7 microRNA family is selectively secreted into the extracellular environment via exosomes in a metastatic gastric cancer cell line. PLoS One. 2010;5(10):e13247.

36. Pigati L, Yaddanapudi SC, lyengar R, Kim DJ, Hearn SA, Danforth D, Hastings ML, Duelli DM. Selective release of microRNA species from normal and malignant mammary epithelial cells. PLoS One. 2010;5(10):-e13515.

37. Wang K, Zhang S, Weber J, Baxter D, Galas DJ. Export of microRNAs and microRNA-protective protein by mammalian cells. Nucleic Acids Res. 2010; 38(20):7248-59.

38. Cookson VJ, Bentley MA, Hogan BV, Horgan K, Hayward BE, Hazelwood LD, Hughes TA. Circulating microRNA profiles reflect the presence of breast tumours but not the profiles of microRNAs within the tumours. Cell Oncol. 2012:35(4):301-8.

39. Cancer Genome Atlas N. Comprehensive molecular portraits of human breast tumours. Nature. 2012:490(7418):61-70.

40. Albeshan SM, Mackey MG, Hossain SZ, Alfuraih AA, Brennan PC. Breast cancer epidemiology in gulf cooperation council countries: a regional and international comparison. Clin Breast Cancer. 2017;

41. Leidner RS, Li L, Thompson CL. Dampening enthusiasm for circulating microRNA in breast cancer. PLoS One. 2013;8(3):e57841.

42. Chen $X$, Liang $H$, Zhang J, Zen $K$, Zhang CY. Secreted microRNAs: a new form of intercellular communication. Trends Cell Biol. 2012;22(3):125-32.

43. Mathe A, Scott RJ, Avery-Kiejda KA. miRNAs and other epigenetic changes as biomarkers in triple negative breast cancer. Int J Mol Sci. 2015;16(12): 28347-76.

44. Li Q, Liu M, Ma F, Luo Y, Cai R, Wang L, Xu N, Xu B. Circulating miR-19a and miR-205 in serum may predict the sensitivity of luminal a subtype of breast cancer patients to neoadjuvant chemotherapy with epirubicin plus paclitaxel. PLoS One. 2014;9(8):e104870

45. Toyama $T$, Kondo $N$, Endo $Y$, Sugiura H, Yoshimoto N, Iwasa M, Takahashi S, Fujii $Y$, Yamashita $H$. High expression of microRNA-210 is an independent factor indicating a poor prognosis in Japanese triple-negative breast cancer patients. Jpn J Clin Oncol. 2012;42(4):256-63.

46. Ghosh Z, Chakrabarti J, Mallick B. miRNomics-the bioinformatics of microRNA genes. Biochem Biophys Res Commun. 2007;363(1):6-11. 
47. Bandi N, Zbinden S, Gugger M, Arnold M, Kocher V, Hasan L, Kappeler A, Brunner T, Vassella E. miR-15a and miR-16 are implicated in cell cycle regulation in a Rb-dependent manner and are frequently deleted or downregulated in non-small cell lung cancer. Cancer Res. 2009;69(13):5553-9.

48. Rekker K, Saare M, Roost AM, Salumets A, Peters M. Circulating microRNA profile throughout the menstrual cycle. PLoS One. 2013;8(11):e81166.

49. Kosaka N, Yoshioka Y, Hagiwara K, Tominaga N, Katsuda T, Ochiya T. Trash or treasure: extracellular microRNAs and cell-to-cell communication. Front Genet. 2013;4:173.

50. Falcone G, Felsani A, D'Agnano I. Signaling by exosomal microRNAs in cancer. J Exp Clin Cancer Res. 2015;34:32.

51. Boyerinas B, Park SM, Hau A, Murmann AE, Peter ME. The role of let-7 in cell differentiation and cancer. Endocr Relat Cancer. 2010;17(1):F19-36.

52. Heneghan HM, Miller N, Lowery AJ, Sweeney KJ, Newell J, Kerin MJ. Circulating microRNAs as novel minimally invasive biomarkers for breast cancer. Ann Surg. 2010;251(3):499-505.

53. Thakur S, Grover RK, Gupta S, Yadav AK, Das BC. Identification of specific miRNA signature in paired sera and tissue samples of Indian women with triple negative breast cancer. PLoS One. 2016;11(7):e0158946.

54. Chen B, Li H, Zeng X, Yang P, Liu X, Zhao X, Liang S. Roles of microRNA on cancer cell metabolism. J Transl Med. 2012;10:228.

55. Singh R, Yadav V, Kumar S, Saini N. MicroRNA-195 inhibits proliferation, invasion and metastasis in breast cancer cells by targeting FASN, HMGCR, ACACA and CYP27B1. Sci Rep. 2015;5:17454.

56. Wang J, Zhang KY, Liu SM, Sen S. Tumor-associated circulating microRNAs as biomarkers of cancer. Molecules. 2014;19(2):1912-38.

57. Heneghan HM, Miller N, Kerin MJ. Circulating microRNAs: promising breast cancer biomarkers. Breast Cancer Res. 2011;13(1):402. author reply 403

58. Bovell LC, Putcha BD, Samuel T, Manne U. Clinical implications of microRNAs in cancer. Biotech Histochem. 2013;88(7):388-96.

59. Heneghan HM, Miller N, Kelly R, Newell J, Kerin MJ. Systemic miRNA-195 differentiates breast cancer from other malignancies and is a potential biomarker for detecting noninvasive and early stage disease. Oncologist. 2010;15(7):673-82.

60. Cecene G, Ak S, Eskiler GG, Demirdogen E, Erturk E, Gokgoz S, Polatkan V Egeli U, Tunca B, Tezcan G, et al. Circulating miR-195 as a therapeutic biomarker in Turkish breast cancer patients. Asian Pac J Cancer Prev. 2016; 17(9):4241-6.

\section{Submit your next manuscript to BioMed Central and we will help you at every step:}

- We accept pre-submission inquiries

- Our selector tool helps you to find the most relevant journal

- We provide round the clock customer support

- Convenient online submission

- Thorough peer review

- Inclusion in PubMed and all major indexing services

- Maximum visibility for your research

Submit your manuscript at www.biomedcentral.com/submit 